УДК 629.7.018

DOI: $10.15827 / 0236-235 X .130 .336-342$

\title{
Требования к программной среде автоматизированной обработки полетных данных
}

\author{
E.M. Еремин 1, инженер-испытатель, јепуа.eryomin2014@yandex.ru \\ А.В. Нагорнов ${ }^{1}$, зам. начальника отдела, начальник отделения, \\ alexnagornov77@bk.ru
} 1 Государственный летно-испытательный иентр им. В.П. Чкалова,
2. Щелково, 141110 , Россия

С середины прошлого века в испытаниях летательных аппаратов, а также в летных исследованиях и полунатурных экспериментах по авиационной тематике существует задача автоматизированной обработки табличных временных рядов экспериментальных данных. Современные программные средства обработки данных обеспечивают успешное решение задач анализа полетной информации, но универсальной программной среды для этих целей не существует: авиационные специалисты сталкиваются с необходимостью использования нескольких разных приложений, что замедляет работу. Этим обусловлена актуальность задачи создания специализированной программной среды, ориентированной на потребности авиационной тематики.

Для формирования требований к новой программной среде рассмотрены типовые элементы работы с полетной информацией: фильтрация данных по заданным критериям, визуализация динамики процесса на графике зависимости параметра от времени, добавление производных временных рядов, визуализация данных на графике зависимости параметра от другого параметра, определение значения обобщающего расчетного показателя на основе всей записи или ее фрагмента, определение взаимосвязей между параметрами, построение моделей множественной линейной и нелинейной регрессии.

Для каждого из типовых элементов приведен пример из практической работы с результатами выполнения экспериментов. Также приведены примеры существующих приложений, решающих конкретные задачи с приемлемым или максимально возможным качеством.

Рассмотрена общая архитектура системы. Предложены использование схемы «модель-вид-контроллер», разработка на языке программирования $\mathrm{C}++$ с использованием паттернов объектно-ориентированного программирования «наблюдатель», «компоновщик» и «стратегия», а также код основы класса Row, каждый объект которого содержит один столбец таблицы данных. Количественные данные столбца таблицы в объекте класса Row размещаются в стандартном контейнере C++ vector, coдержащем элементы типа double.

Ключевые слова: полетная информация, обработка экспериментальных данных, сглаживание временных рядов, построение графиков, регрессионные модели, требования к программной продукции, архитектура приложения.

Важнейшей составляющей жизненного цикла программного продукта является этап, предшествующий написанию кода. При этом одна из главных проблем - требования к будущему приложению: ошибки, допускаемые при их выявлении и формализации, имеют, с одной стороны, высокую цену устранения, а с другой - большую частоту встречаемости в реальных проектах [1-3].

В статье рассмотрена работа с регистрируемыми значениями параметров, прямо или косвенно относящихся к описанию динамики полета летательного аппарата (ЛА). Такие данные записываются системами бортовых измерений (СБИ), системами объективного контроля ЛА и некоторыми другими. Результатом записи является таблица, содержащая вре-

менные ряды (функцию зависимости от времени) по каждому из параметров.

Автоматизированная обработка табличных временных рядов осуществляется в испытаниях ЛА, летных и полунатурных исследованиях с середины прошлого века.

Многие десятилетия размер исходных данных практически не меняется: ориентировочный объем записи летного эксперимента начала 1960-х гг. (до 100 столбцов и 180 тыс. строк [4]) актуален и в настоящее время. При этом принципиально выросли возможности извлечения полезной информации из экспериментальных данных [5], что обусловлено ростом производительности и памяти вычислительной техники в совокупности с улучшением эргономики компьютеров и их программ. 
Сегодня важнейшую роль в обработке данных играют возможности используемой специалистом программной среды. Большинство современных инструментов реализуют идеологию WYSIWYG (сокращение от what you see is what you get, или что ты видишь, то и получаешь). Существуют мощные программные продукты, обеспечивающие простой и удобный для пользователя вызов разнообразных встроенных алгоритмов обработки данных. Эти возможности совмещены с поэлементными настройками отображения данных на графиках, вводом данных из файлов различных форматов и многими другими полезными опциями.

Обработка полетной информации состоит из довольно специфичного набора действий с данными. При всех достоинствах современных сред обработки информации ни одна из них не является хорошим решением для всего перечня задач. Подобная ситуация не уникальна. Существование общей проблемы использования унифицированного ПО в организациях научноисследовательского профиля отмечено в исследовании специалистов Иркутского национального исследовательского технического университета [6]. В качестве иллюстрации этого тезиса можно рассмотреть пример обработки информации в химико-технологической системе [7]: для решения практической задачи потребовалось использование четырех программных сред - TableCurve 2D, TableCurve 3D, MATLAB и Enterprise Workbench 2k. реализации не заменит удачную формулировку желаемых свойств системы, но послужит их наглядной иллюстрацией.

Рассмотрим более детально конкретные задачи обработки полетных данных.

Фильтрация данных таблицы по заданному критерию. Данная задача позволяет определять строки, для которых вектор мгновенного состояния системы (режим полета ЛА) соответствует заданным условиям.

Пример: поиск режимов полета, соответствующих висению вертолета вне зоны влияния земли. Задача решается проверкой составного условия по параметрам, отражающим геометрическую высоту (данные радиовысотомера) и составляющие вектора скорости (данные внешнетраекторных измерений). Уточнение задачи (например, висение вне зоны влияния земли правым боком к ветру с заданным качеством стабилизации) добавляет новые условия фильтрации.

Существующие приложения: MS Excel.

Визуализация динамики процесса на графике зависимости параметра от времени. Пример: построение графика изменения параметров полета ЛА после выведения его из состояния сбалансированного полета импульсным управляющим воздействием. На рисунках 1 и 2 приведены записи режимов «импульс ручкой циклического шага», выполненных на вертолете Ка-52Э в ходе летного эксперимента. Ручка отклонялась летчиком соответственно от

Стоит отметить, что большинство отдельно взятых задач обработки полетной информации имеют хорошее решение минимум в одном из существующих приложений.

Идеальная программная среда должна объединять в себе определенные возможности нескольких уже существующих систем. Это важно с точки зрения проблемы формирования требований: наличие готового примера

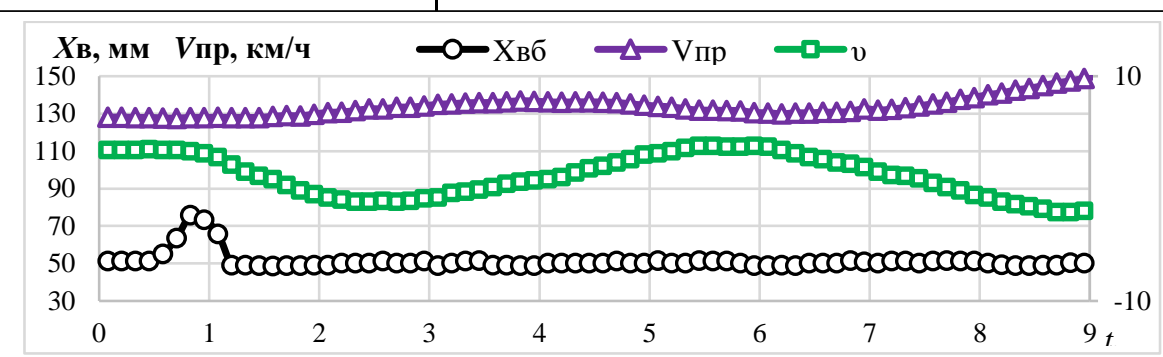

Рис. 1. Импульс ручкой управления от себя, реакция вертолета

Fig. 1. Central stick push impulse, helicopter reaction

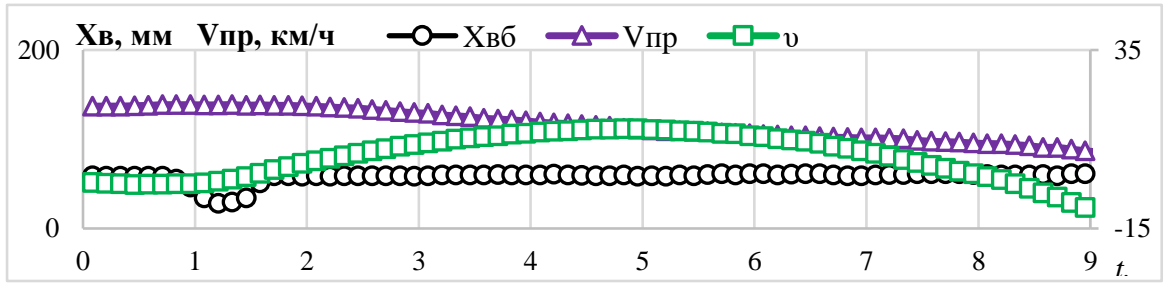

Рис. 2. Импульс ручкой управления на себя, реакция вертолета

Fig. 2. Central stick pull impulse, helicopter reaction 
себя и на себя (положение ручки характеризуется величиной $X$ в). Визуализация позволяет оценить поведение вертолета в первые секунды свободного движения. Здесь показаны прибор-

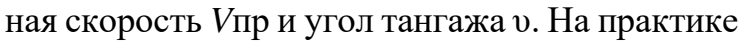
рассматриваются и другие регистрируемые и расчетные параметры.

Существующие приложения: РTC MathCad, Ivan Johansen Graph, Alentum Software Advanced Grapher, FBK Studio Software Grapher.

Добавление производных временных рядов (сглаживающие, интегрирующие, дифференцирующие ряды, ряды расчетных значений физических величин, не регистрировавшихся СБИ или нуждающихся в проверке точности регистрации).

Пример: добавление к данным рисунка 2 расчетных значений угловой скорости движения вертолета в продольном канале (рис. 3).

Существующие приложения: интерполяция и аппроксимация - Systat Software TableCurve 2D, ряды расчетных значений по формулам пользователя - MS Excel.

Визуализация данных на графике зависимости параметра от другого параметра. Пример: проверка корректности регистрируемых значений параметра построением функциональной зависимости регистрируемого и расчетного значений. На рисунке 4 показана проверка регистрации СБИ самолета Да-42Т значений угла атаки $(\alpha)$. Положительный результат проверки соответствует группированию облака точек вблизи линии $y=x$ (значение первого коэффициента уравнения регрессии близко к 1, второго к 0, коэффициента детерминации - к 1). Очевидно, что представленные данные иллюстрируют некорректную работу СБИ.

Существующие приложения: РТC
MathCad, Ivan Johansen Graph, FBK Studio Software Grapher.

Определение значения обобщающцего расчетного показателя на основе всей записи или ее фрагмента (режима). Пример: определение балансировочного положения органа управления для заданных условий по данным выполнения режима «площадка». Режим характеризуется постоянством скорости, высоты и углового положения вертолета (все силы и моменты, действующие на вертолет, скомпенсированы). На рисунке 5 приведена одна из балансировочных характеристик вертолета Ка-52Э, построенная по результатам испытаний. Значение параметра Хв для каждой точки графика получено обработкой записи отдельного режима полета.

Существующие приложения: MS Excel, PTC MathCad.

Определение взаимосвязей между параметрами, построение моделей множественной линейной и нелинейной регрессии. Пример: построение модели движения вертолета в продольном канале путем вычисления значений коэффициентов дифференциального уравнения движения вертолета (коэффициенты

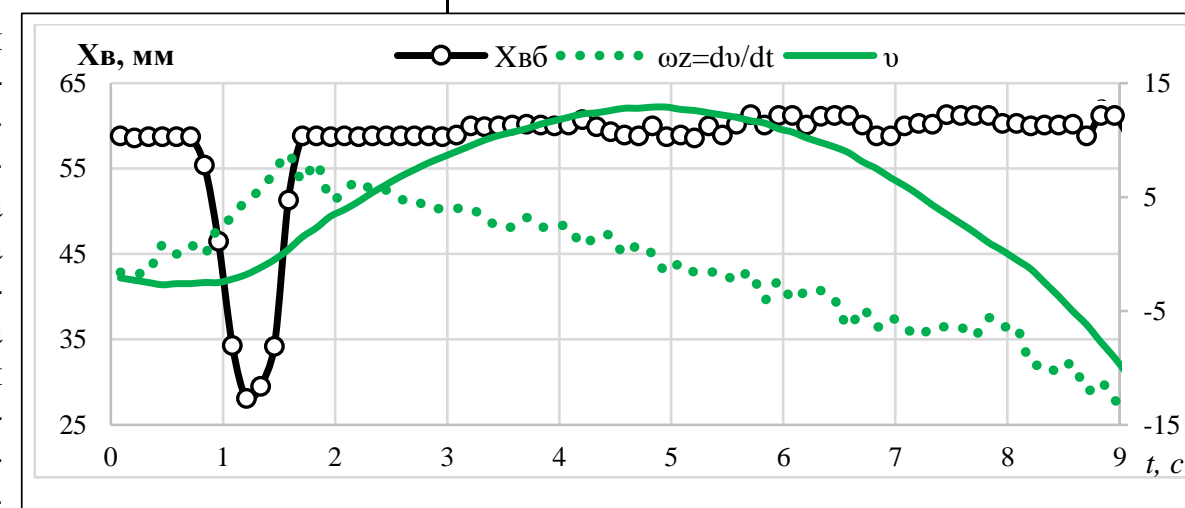

Рис. 3. Численное дифференцирование ряда «угол тангажа» по времени

Fig. 3. Numerical differentiation of the "pitch angle" series in time

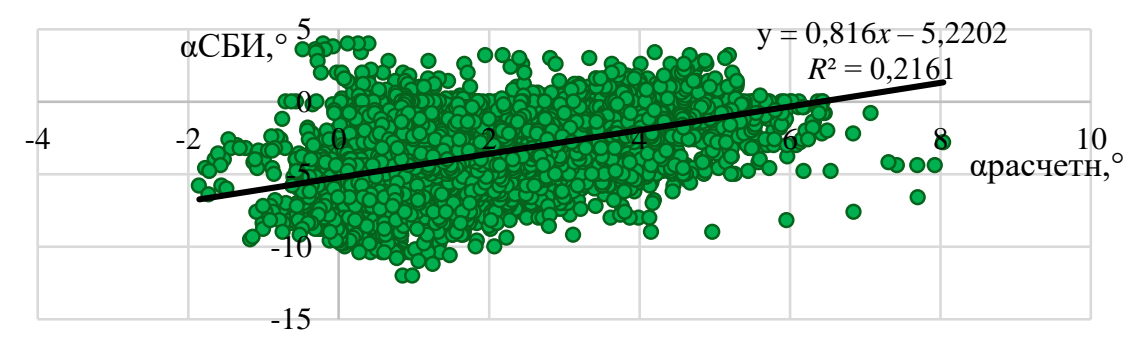

Рис. 4. Проверка корректности работы системы бортовых измерений, регистрация угла атаки

Fig. 4. The onboard measurement system checking, attack angle registration 
демпфирования, эффективности управления и устойчивости по скорости).

На рисунке 6 приведены данные для оценки адекватности модели движения вертолета Ка$52 Э$ в продольном канале, полученной на основе множественной линейной регрессии по массиву из нескольких десятков тысяч точек. Очевидно, что исследуемая модель не демонстрирует высокое качество, воспроизводя с высокой точностью лишь первую реакцию на интенсивное возмущение входного сигнала. Для повышения точности модели требуется усложнение ее вида.

Существующие приложения: множественная линейная регрессия для произвольного числа аргументов - Dell Statistica или MS Excel c расширяющим пакетом «Анализ данных», множественная нелинейная регрессия для функции двух аргументов - Systat Software TableCurve 3D, множественная нелинейная регрессия с числом аргументов более двух требует написания дополнительного кода для расширения готовых программных продуктов, например, на языке visual basic for applications для MS Excel.

Приведенный перечень представляет основные функциональные требования к программной среде обработки полетных данных.

Список требований, отталкивающихся от достоинств существующих систем, следует дополнить списком требований, отталкивающихся от их недостатков:

- программная среда должна работать с файлами максимального размера (ограничения приведены выше);

- программная среда должна обеспечивать быстрое (насколько позволяет аппаратная со- ставляющая) выполнение вычислений;

- взаимодействие пользователя с графическим интерфейсом должно быть построено в соответствии с эргономическим принципом «наименьшего удивления».

Важнейшим требованием к создаваемой системе следует считать приспособленность к итерационному наращиванию функционала. Ни один список требований не может считаться исчерпывающим, поскольку в перспективе неизбежно появятся новые потребности, например, по использованию искусственных нейронных сетей. Для обеспечения простоты доработок архитектура системы должна быть модульной. В ее основе следует использовать решения, показавшие эффективность в управлении сложностью кода.

Исходя из имеющегося перечня требований, целесообразно для реализации системы использовать язык программирования $\mathrm{C}++$. Этот язык является одним из лучших по быстродействию и экономии памяти [8], что имеет важное значение, например, для решения ре- 
грессионных задач с большими входными данными на компьютерах, не обладающих высокими техническими характеристиками. Кроме того, он поддерживает объектно-ориентированное программирование с его концепциями, обеспечивающими модульность и повторное использование кода [9]. С++ поддерживает применение набора проверенных решений в организации кода - паттернов проектирования. В частности, для создания приложений рассматриваемой категории применяется архитектура «модель-вид-контроллер», реализуемая применением паттернов «наблюдатель», «компоновщик» и «стратегия» [10].

Программное решение для размещения данных (компонента «модель») следует искать прежде всего среди стандартных контейнеров $\mathrm{C}++$. Наиболее подходящим из них представляется vector. Размещением данных каждого ряда внутри своего объекта vector обеспечивает, с одной стороны, гибкость в использовании памяти, а с другой, высокую скорость случайного (произвольного) доступа [11]. Максимальное количество элементов ряда при использовании контейнера vector ограничивается только памятью компьютера.

Сами ряды целесообразно реализовать в виде объектов пользовательского класса, основа которого показана в листинге:

\#include <vector> //для использования контейнepa vector

\#include <string> //для использования строк string

class Row

\{

public:

//настройка названия ряда:

void setName (string paramName);

//присвоение значения val элементу ряда с номером itemNum:

void setValue (double val, int itemNum);

//добавление нового элемента со значением val в конец ряда:

void addValue (double val);

//функции выдачи названия ряда и значения его элемента по номеру:

string getName ();

double getValue (int numberOfVal);

/*3десь находятся другие необходимые функции, в том числе конструкторы и деструктор*/ protected:

string name;

vector <double> data; //ряд данных - веществен-

ных чисел

\};

Для поля Row::data выбран тип double, поскольку подавляющее большинство регистрируемых параметров имеют вещественный тип. Целочисленные данные, включая логические «0» и «1», встречаются гораздо реже, поэтому случаи неявного приведения типов не окажут существенного влияния на экономию памяти.

Дополнительная открытая функция Row::addValue(), изменяющая поле Row::data, предназначена для вызова внешним кодом при заполнении пустого ряда значениями. Пример такой ситуации - заполнение значениями, считываемыми построчно из файла исходных данных.

Выбор контейнера для размещения самих объектов класса Row в меньшей степени влияет на эффективность программы, поскольку количество этих объектов (столбцов таблицы) обычно на два-три порядка меньше количества элементов поля Row::data (количества строк). Если в случае реализации поля Row::data определяющим фактором является скорость случайного доступа к элементам данных, то для контейнера, вмещающего сами объекты класса Row, может быть важна также скорость вставки и удаления элементов, что делает предпочтительным шаблон list. Окончательное peшение должно быть принято по результатам проработки альтернативных вариантов.

\section{Заключение}

На основе практического опыта анализа полетной информации сформулированы требования к программной среде автоматизированной обработки полетных данных в терминах предметной области. Задачи создаваемой системы соотнесены с примерами существующих систем, подтвердивших свою практическую применимость.

Составленный список общих задач системы следует использовать для выработки требований в терминах программирования и их реализации в коде проекта. Приведенные рекомендации целесообразно использовать в архитектуре проекта.

\section{Лuтература}

1. Гутгарц Р.Д., Провилков Е.И. О формализации функциональных требований в проектах по созданию информационных систем // Программные продукты и системы. 2019. № 3. С. 349-357. DOI: 10.15827/0236- 235X.127.349-357. 
2. Wiegers K., Beatty J. Software Requirements. USA, Microsoft Press, 2013, 637 p.

3. Макконнелл С. Совершенный код. Практическое руководство по разработке программного обеспечения. М., 2010. 896 с.

4. Кантор А.В. Аппаратура и методы измерений при испытаниях ракет. М., 1963.520 с.

5. Еремин Е.М., Атрошенко А.И., Антошин А.А., Дроздовский А.Ю. Определение пилотажных характеристик модели вертолета авиационного тренажера КТ-8АМТШ // Проблемы безопасности полетов. 2019. № 1. С. 10-17.

6. Гутгарц Р.Д., Полякова П.М. Анализ особенностей формулирования функциональных требований к автоматизированной информационной системе // Программные продукты и системы. 2019. № 3 . C. 358-367.

7. Кузнецов А.С., Корнюшко В.Ф. Математические модели реограмм состояния в программах Table Curve 2D/3D как основа интеллектуальной системы управления процессами структурирования многокомпонентных эластомерных композитов // Программные продукты и системы. 2017. № 4 . C. $770-777$. DOI: $10.15827 / 0236-235 X .120 .0$

8. Pereira R., Couto M., Ribeiro F., Rua R., Cunha J., Fernandes J.P., Saraiva J. Energy efficiency across programming languages. How do energy, time, and memory relate. Proc. 10th ACM SIGPLAN Intern. Conf. on SLE, Vancouver, Canada, 2017, pp. 256-267.

9. Спролл А. Думай как программист: креативный подход к созданию кода. С++ версия. М., 2018. $272 \mathrm{c}$.

10. Гамма Э., Хелм Р., Джонсон Р., Влиссидес Д. Приемы объектно-ориентированного проектирования. Паттерны проектирования. СПб, 2019. 368 с.

11. Лафоре Р. Объектно-ориентированное программирование в С++. СПб, 2019. 928 с.

\title{
Requirements for the software environment of automated flight data analysis
}

\author{
E.M. Eremin ${ }^{1}$, Test Engineer, jenya.eryomin2014@yandex.ru \\ A.V. Nagornov ', Deputy Head of Department - Branch Manager, alexnagornov77@bk.ru \\ ${ }^{1}$ Chkalov State Flight Test Center, Shchelkovo, 141110, Russian Federation
}

\begin{abstract}
In aircraft tests, as well as in flight researches and semi-natural experiments on aviation issues since the middle of the last century, there is an automated processing problem for the tabular time series of experimental data. Modern data analysis software provides a solution to the flight information analysis problems, but a universal software environment for these purposes does not exist: aviation professionals encounter with the necessity to use several different applications, which slows down the work. An urgent problem is to create a specialized software environment focused on aviation topic needs.

To produce the requirements for the new software environment, there are the working typical elements with flight information: data filtering according to specified criteria, process dynamics visualization on the graph of parameter dependence on time, time-series derivatives addition, data visualization on the graph of parameter dependence on another parameter, determination of the generalizing calculated indicator value on the entire record or its fragment basis, relationship determination between parameters, multiple linear constructions and nonlinear regression models.

For each of the standard elements, there is a practical work example with the performing experiment results. There are also existing application examples that solve specific problems with acceptable or maximum possible quality.

This paper contains the system general architecture. The authors proposed the use of the model-viewcontroller scheme, development in the $\mathrm{C}++$ programming language using the object-oriented programming patterns observer, linker and strategy, as well as the Row class basic code, each object of which contains one data table column. The quantitative data of a table column in an object of the Row class is in the standard C++ vector container, which contains double type elements.
\end{abstract}


Keywords: flight information, experimental data analysis, time series smoothing, plotting, regression models, software requirements, application architecture.

\section{References}

1. Gutgarts R.D., Provilkov E.I. On the formalization of functional requirements in information system projects. Software \& Systems, 2019, vol. 32, no. 3, pp. 349-357 (in Russ.). DOI: 10.15827/0236- 235X.127. 349-357.

2. Wiegers K., Beatty J. Software Requirements. USA, Microsoft Press, 2013, 637 p.

3. McConnell S. Code Complete: A Practical Handbook of Software Construction. USA, Washington: Microsoft Press, 2004, 960 p. (Russ. ed.: Moscow, 2010, 896 p.).

4. Kantor A.V. Instrumentation and Measurement Methods for Missile Tests. Moscow, 1963, 520 p. (in Russ.).

5. Eremin E.M., Atroshenko A.I., Antoshin A.A., Drozdovsky A.Yu. Determination of flight characteristics of the flight simulator KT-8AMTSH helicopter model. Flights Safety Troubles, 2019, no. 1, pp. 10-17 (in Russ.).

6. Gutgarts R.D., Polyakova P.M. Analysis of formulation features of functional requirements to an automated information system. Software \& Systems, 2019, vol. 32, no. 3, pp. 358-367 (in Russ.).

7. Kuznetsov A.S., Kornyushko V.F. Mathematical models of rheograms of states in Table Curve 2d/3d programs as a basis of the intelligent system for managing structuring processes of multicomponent elastomer composites. Software \& Systems, 2017, vol. 30, no. 4, pp. 770-777 (in Russ.). DOI: 10.15827/0236235X.120.0.

8. Pereira R., Couto M., Ribeiro F., Rua R., Cunha J., Fernandes J.P., Saraiva J. Energy efficiency across programming languages. How do energy, time, and memory relate. Proc. 10th ACM SIGPLAN Intern. Conf. on SLE, Vancouver, Canada, 2017, pp. 256-267.

9. Spraul A. Think Like a Programmer: An Introduction to Creative Problem Solving. Edwards Brothers Malloy Publ., 2012, 315 p. (Russ. ed.: Moscow, 2018, 272 p.).

10. Gamma E., Helm R., Johnson R., Vlissides J. Design Patterns. Elements of Reusable Object-Oriented Software. Addison-Wesley Publ., 1995, 395 p. (Russ. ed.: St. Petersburg, 2019, 368 p.).

11.Lafore R. Object-Oriented Programming in C++. Indianapolis, Sams Publ., 2002, 1040 p. (Russ. ed.: St. Petersburg, 2019, 928 p.).

\section{Для цитирования}

Еремин Е.М., Нагорнов А.В. Требования к программной среде автоматизированной обработки полетных данных // Программные продукты и системы. 2020. Т. 33. № 2. С. 336-342. DOI: $10.15827 / 0236-235 X .130 .336-342$.

\section{For citation}

Eremin E.M., Nagornov A.V. Requirements for the software environment of automated flight data analysis. Software \& Systems, 2020, vol. 33, no. 2, pp. 336-342 (in Russ.). DOI: 10.15827/0236235X.130.336-342. 